\title{
REFERENCES
}

Alonso, E. E. (1976). Probabilidad y geotecnia (segunda parte). Revta Obr. públ., Sept., 753-762.

Eden, W. J. (1967). Buried soil profile under apron of an earthflow. Geological Society of America Bull. 78, 1183-1184.

Eden, W. J. \& Mitchell, R. J. (1973). Landslides in sensitive marine clay in Eastern Canada. HRB Record, No. 463, 18-27, Washington: Highway Research Board.

Jarrer, P. M. \& Eden, W. J. (1970). Groundwater flow in Eastern Ottawa. Technical note. Canad. Geotech. Jnl 7, 326-333.

Lefebvre, G. \& LaRochelle, P. (1974). The analysis of two slope failures in cemented Champlain clays. Can. Geotech. Jnl 11, No. 1, 89-108.

\section{Combined active and passive rotational failure of a retaining wall in sand}

MILligAN, G. W. E. \& BRANSBY, P. L. (1976). Géotechnique 26, No. 3, 473-494.

\section{J. S. Steenfelt, Danish Geotechnical Institute}

With the paper of Milligan and Bransby the Cambridge group has made another valuable contribution to increase the knowledge of the stress-strain behaviour of sand. However, it seems that the theoretical interpretation of the test results is influenced too much by the traditional application of the method of characteristics. Moreover, the assumptions, measurements and interpretations concerning the wall friction $\delta$ leaves the reader rather confused.

In the present contribution a few points will be made about the assumptions and the attention is drawn to the earth pressure theory of Brinch Hansen (1953) which has been shown to be surprisingly accurate in the prediction of earth pressures.

\section{WALL FRICTION}

Apparently the Authors aimed at a smooth wall as the Duralumin was rubbed with successively finer grades of emery paper. It is very doubtful, however, that a Duralumin surface in contact with sand could ever be considered even relatively smooth, as the sand acts as a very efficient coarse emery paper. The recorded wall friction distribution measured by the load cells, which presumably have the same surface as the rest of the wall, bear out that the wall is at any rate relatively rough. The measurements surely leave the Authors in a dilemma as they state that the mobilized value of $\delta$ measured by the authors was about $15^{\circ}$ even though the wall itself was much smoother. Either the value of $\delta$ corresponds to the roughness or the load cell readings are misleading. In this context it is very important to realize that the mobilized angle of wall friction can obtain any value $-\varphi \leq \delta \leq \varphi$ depending only on the state of strain. Even if the sand in contact with the wall behaves like a rigid body the equals signs are allowed (the body is on the point of sliding). Reasonably smooth surfaces might be obtained if the wall and load cells were fitted with a glass cover combined with layers of silicone grease and rubber membranes as is used in triaxial tests with smooth pressure heads.

\section{MODE OF FAILURE}

Two different failure mechanisms were observed: a so-called 'deep failure' for positions of the axis of rotation near the foot of the wall, and 'shallow failure' for higher positions. In my opinion this is merely a question of changes in the original geometry of the tests-an important 


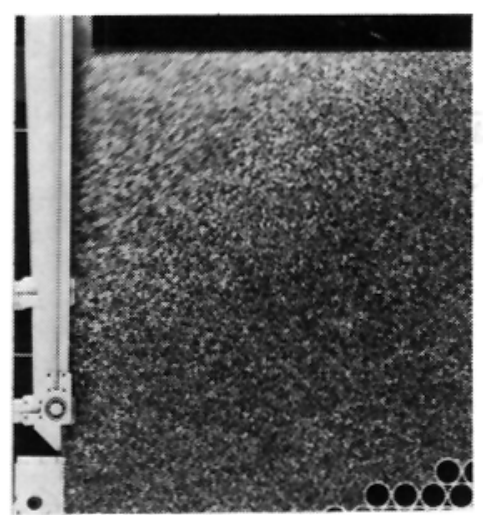

(a)

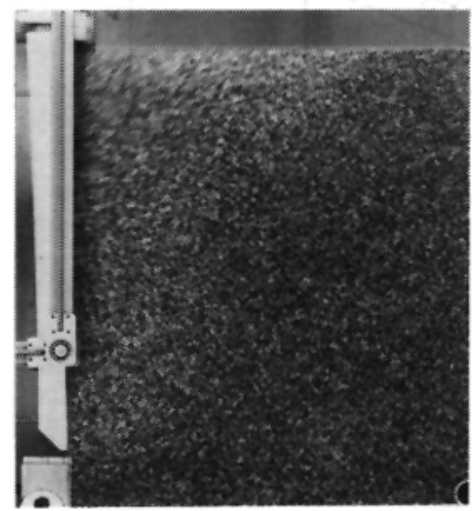

(c)

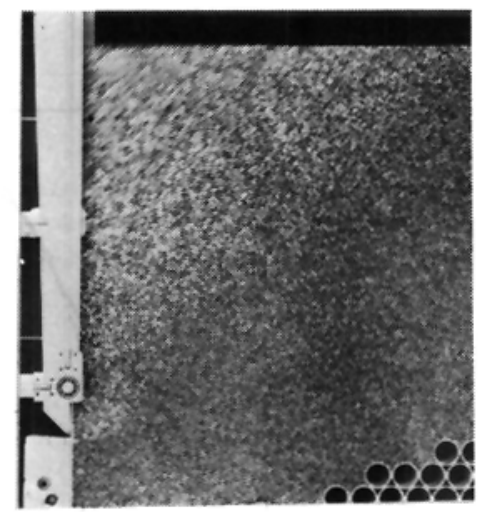

(b)

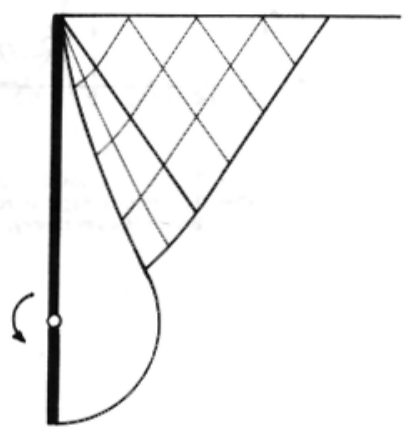

(d)

Fig. 1. Recorded displacement fields for negative rotation of the wall: (a) $\rho=0.11$; (b) $\rho=0.13$; (c) $\rho=0.25$; (d) AaP rupture figure

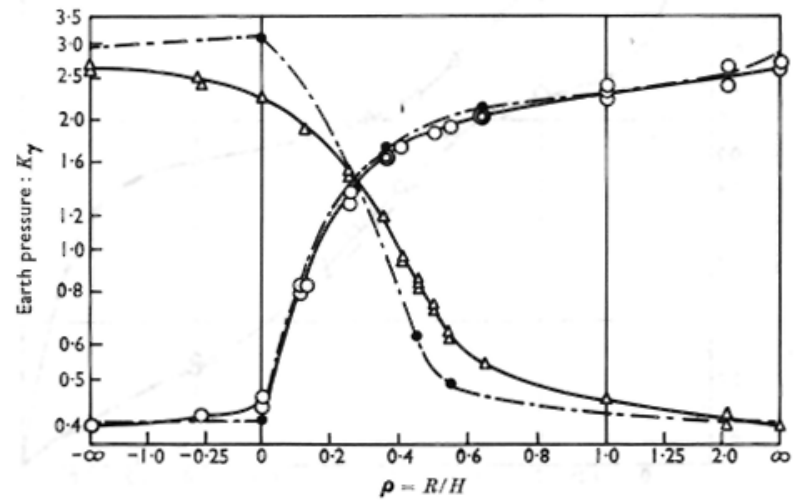

(a)

Fig. 2. Earth pressure coefficients for a rough vertical wall rotating about an axis $\rho=R \mid H$ from the toe (after Hansen and Steenfelt, 1976) 


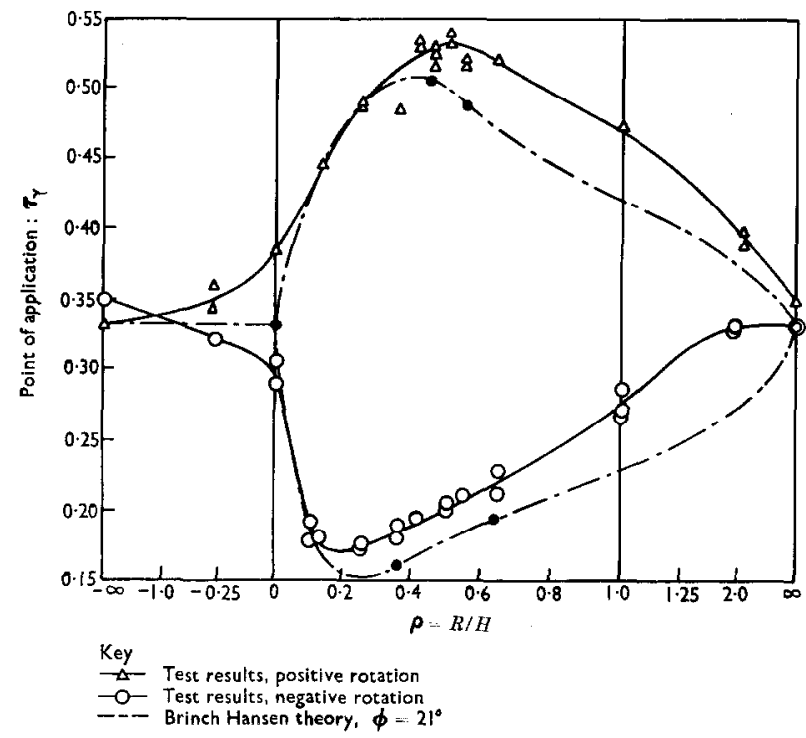

(b)

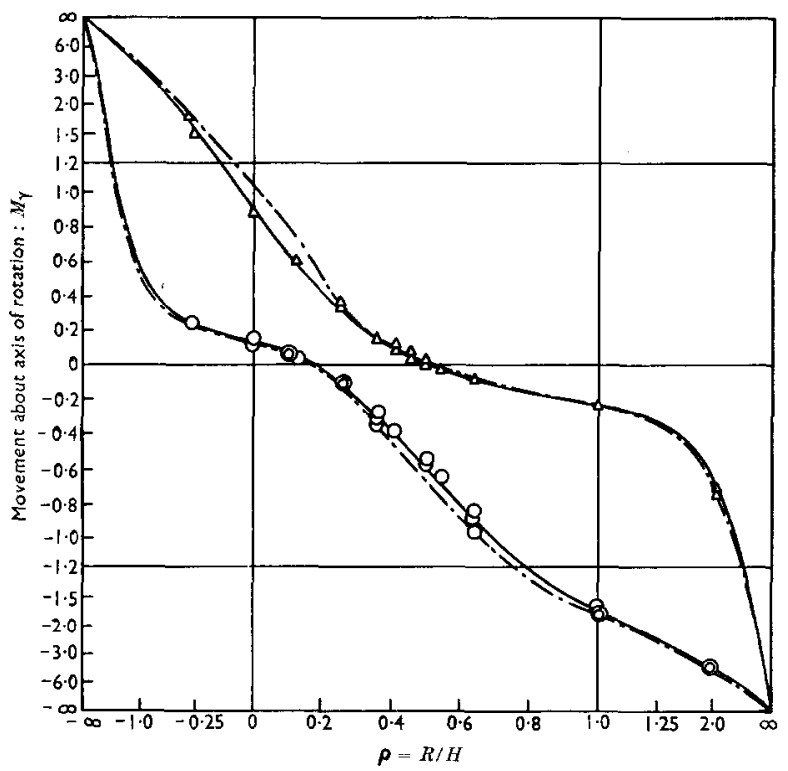

(c)

Fig. 2 (contd) 


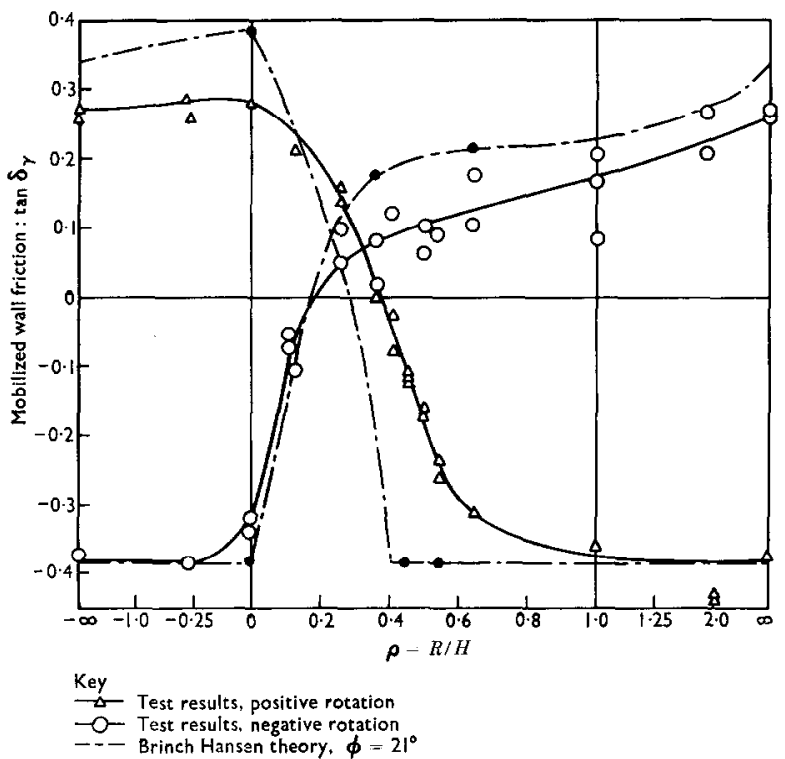

(d)

Fig. 2 (contd)

factor which is often ignored and which leads to misinterpretation of the test results. The magnitude of the changes in geometry (the angle of wall rotation being the most important in the authors' tests) is very closely connected to the definition of the failure. As an example let us consider a passive zone rupture which is supposed to develop on the wall below the axis of rotation. According to Lundgren and Brinch Hansen (1958) the earth pressure coefficient (normal component) for a rough wall inclined at an angle $\theta$ (positive as defined by the Authors) relative to a vertical wall is

$$
\frac{K_{\gamma}{ }^{r}(\theta)}{K_{\gamma}{ }^{r}(\theta=0)} \approx \cos \theta \exp (2 \theta \tan \varphi)
$$

If the passive force and the passive moment in the Authors' Fig. 17 are corrected for the above qualitative increase due to the increase in $\theta$ a value of $\theta \approx 2^{\circ}-3^{\circ}$ at failure would result. (A similar qualitative evaluation of passive earth pressure tests published by the Cambridge group gives a better-defined state of failure for smaller values of the wall movements.) As stated by the Authors, the failure above the axis of rotation is developed almost instantaneously. When $\theta$ is increased to $2^{\circ}$ to $3^{\circ}$, the state of failure is reached for the problem in question. Further increase of $\theta$ corresponds to failure in an anisotropic heterogeneous deposit with a changed geometry, allowing a slip surface to extend from the toe towards the surface.

\section{EARTH PRESSURE THEORY}

The earth pressures predicted by the method of characteristics do not correspond very well to the test results. If, however, Brinch Hansen's earth pressure theory is applied, it is believed that the correspondence would be much better, and the test results could be referred to a constant $\varphi$ value determined by triaxial tests on samples of the same density as the backfill (either plane strain values or axisymmetric values corrected by the well established formula $\varphi_{\text {plane }}=1 \cdot 1 \varphi_{\text {triaxial }}$. 
In an extensive series of earth pressure tests with a vertical rough wall rotated about axes on the wall proper, or its extensions, I found surprisingly good agreement between predicted and measured earth pressurcs. The tests were performed in a pin model (the two-dimensional mechanical analogy model of sand in plane strain) and are presented by Hansen and Steenfelt (1976).

The basis of the theory is the introduction of composite rupture figures allowing rigid bodies to be contained in the rupture figure. The geometry of the bodies is found by kinematical considerations and, as was fully recognized by Brinch Hansen, the movements in the soil must be compatible with those of the wall. For comparison, recorded displacement fields for values of $\rho=R / H$ close to those of the Authors, are shown in Fig. 1 together with the theoretical statically admissible rupture figure denoted AaP. The domain of failure is somewhat larger than indicated by the rupture figure and, as generally found in my test series, the boundary to the rupture figure is more likely to be a zero extension line than a stress characteristic.

In Fig. 2 the theory is compared with the obtained dimensionless earth pressure coefficients corresponding to:

(a) the normal force

$$
E=\frac{1}{2} \gamma H^{2} K_{y}
$$

(b) distance from the toc of the wall to the point of application

$$
Z=\zeta_{y} H
$$

(c) moment about the axis of rotation

$$
M=E(Z-R)=\frac{1}{2} \gamma H^{3} M \gamma ;
$$

(d) the tangential force (positive for upward movement of the sand relative to the wall; $\delta_{y}$ is the mean mobilized angle of wall friction)

$$
F=E \tan \delta_{\gamma} .
$$

The general agreement is very good, especially for negative rotation which is used in the Authors' test series. Negative rotation means that the angle between wall and surface, measured through the soil, decreases. In this connection it is recommended that the terms positive and negative rotation are used to describe the movements of the wall, as this notation is unambiguous and generally valid. A meaningless use of the terms active and passive failure is thus avoided, as both types of failure take place for most positions of the axis of rotation.

\section{Authors' reply}

We thank Steenfelt for his interest in the Paper and for his opening remarks. Nevertheless, we cannot accept most of his comments about the Paper and will reply to the points in the sequence in which he raises them.

\section{WALL FRICTION}

The quoting of a sentence out of context seems to obscure an important point made in the Paper: the mobilized local angle of wall friction depends not only on the surface roughness of the wall but on the state of stress and strain in the sand adjacent to the wall. Clearly, the mobilized angle of wall friction can never exceed the mobilized angle of internal friction in the sand. Thus, if the sand 'behaves like a rigid body', and the mobilized value of $\varphi$ is well below the peak value of $\varphi$, correspondingly, the mobilized value of $\delta$ must also be well below the peak value of $\delta$. However, even if the mobilized $\varphi$ is equal to the peak value of $\varphi$ for the sand, and 
even if the surface of the wall is rough, there is no guarantee that the mobilized value of $\delta$ on the wall is high. Indeed the data shown in Fig. 19 suggest that the mobilized value of $\delta$ can be zero, even when both conditions are satisfied. The mobilized value of $\delta$ thus depends on the orientation of the principal stresses as well as on the mobilized value of $\varphi$.

To clear up any possible misunderstanding, it should perhaps be added that we did not intend to perform experiments for the unrealistic case when the wall was perfectly smooth.

\section{MODE OF FAILURE}

It is clear from Fig. 6 that different failure mechanisms were observed for tests 24 and 30 . The original radiographs show that there was no sign at $\theta=6^{\circ}$ in test 24 of the mechanism observed at $\theta=6^{\circ}$ in test 30 . Thus 'the changes in the original geometry of the tests' cannot be the factor causing the difference in failure mechanism. It seems clear that the differences in failure mechanism were a result of the differences in $R / H$ between the two tests which were otherwise identical.

Steenfelt is correct in asserting that the theoretical predictions of forces on the wall depend on the inclination of the wall, and therefore that the predicted force for $\theta=3^{\circ}$ is different from the predicted force for $\theta=0^{\circ}$. Nevertheless, it is surely incorrect to adjust the observed values of forces and moments to allow for changes in geometry (i.e. changes of $\theta$ from zero) in order that a mathematical 'definition of failure' can be achieved. For any application, it is required to know what the actual maximum force or moment on the wall will be, and that maximum will be relevant for displacements (of unknown magnitude) from a specified initial condition. In addition, it is perhaps worth noting that conventional methematical predictions are made for the unrealistic case where the mobilized value of $\varphi$ is everywhere equal to the peak value of $\varphi$. Such analyses can take no account of the differences in forces on the wall which would be observed at $\theta=3^{\circ}$ in two experiments usuing dense sand which started from the initial conditions of (i) $\theta=0^{\circ}$ and (ii) $\theta=-10^{\circ}$, even if the geometrical conditions (especially at the surface of the sand) were arranged to be identical in the two tests.

\section{EARTH PRESSURE THEORY}

Steenfelt's results of his experiments are of considerable interest; and the comparisons with Brinch Hansen's earth pressure theory strikingly good. Perhaps his data would have been of even more interest had he used sand rather than a mechanical analogue of sand and had he used the stereo-photogrammetric technique of Butterfield et al. (1970) to give the magnitudes of the displacements. The reasons why the method of characteristics gave good predictions for some regions of the sand and less good predictions for other regions was discussed in the Paper. It is believed that the major part of the discrepancies arise because of the assumption of a constant value of $\varphi$ everywhere in the sand, an assumption that is also made in Brinch Hansen's method. One method for overcoming this deficiency, the method of Serrano (1972) and James et al. (1972) was referred to in the Paper.

\section{REFERENCES}

Brinch Hansen, J. (1953). Earth pressure calculation. Copenhagen: Danish Technical Press.

Butterfield, R., Harkness, R. M. \& Andrawes, K. Z. (1970). A stereo-photogrammetric method for measuring displacement fields. Géotechnique 20, No. 3, 308-314.

Hansen, B. \& Steenfelt, J. S. (1976). J. Brinch Hansen's earth pressure theory tested by experiments in a pin model. Proc. 5th Budapest Conf. Soil Mech., Budapest, 447-458.

Lundgren, H. \& Brinch Hansen, J. (1958). Geoteknik (Soil Mechanics). Copenhagen: Danish Technical Press. 